Precaución de contacto, error médico y eventos adversos Impact of contact isolation for multidrug-resistant organisms on the occurrence of medical errors and adverse events.

Zahar $J R$, Garrouste-Orgeas $M$, Vesin A, Schwebel C, Bonadona A, Philippart F, et al. Intensive Care Med 2013; 39: 2153-60.
La precaución de contacto, son una medida efectiva ampliamente utilizada en la prevención de la transmisión cruzada de microorganismos multirresistentes. Sin embargo, en los últimos años se ha asociado a resultados negativos inesperados en los pacientes. El objetivo de este estudio fue comparar la ocurrencia de eventos adversos en pacientes con precaución de contacto versus sin precaución, en Unidades de Paciente Crítico (UPC).

Método: Ensayo controlado, multicéntrico, en el que se incluyeron pacientes adultos hospitalizados en UPC en cuatro períodos, entre enero de 2007 y enero de 2008. El objetivo fue evaluar la efectividad de tres programas de seguridad de pacientes y comparar la ocurrencia de eventos adversos en pacientes con precaución de contacto versus pacientes sin aislamiento. Se excluyeron los pacientes con neutropenia o trasplantados de órganos sólidos.

Resultados: Se incluyeron 1.150 pacientes de una UPC Médica, de 18 camas, y una Médico Quirúrgica, de 10 camas. La relación enfermera: paciente estimada era de 2,8: 2,5; respectivamente, sin embargo, este dato no fue recolectado en el estudio. Ciento setenta pacientes $(14,8 \%)$ estaban con precaución de contacto. La edad promedio fue de 62 años $( \pm 17)$, con índice pronóstico SAPS promedio $43( \pm 21)$. El diagnóstico más frecuente en el grupo de pacientes aislados fue falla respiratoria o multi-orgánica. De los 170 pacientes con precaución de contacto, $75(44,1 \%)$ presentaron eventos adversos antes del inicio del aislamiento y $140(82,4 \%)$, durante el período de aislamiento. La incidencia global de eventos adversos fue mayor durante el período de aislamiento, resultando significativos la neumonía asociada a ventilación mecánica (NAV), errores en prescripción, administración de anticoagulantes, hipo o hiperglicemia e hipercalcemia.

Discusión: En este estudio, la precaución de contacto se asoció significativamente a un aumento de eventos adversos; sin embargo, no se evaluó si tuvieron relación con la gravedad del paciente o fueron una consecuencia directa del aislamiento. Otras publicaciones también han reportado que esta medida puede tener consecuencias deletéreas para los pacientes, tales como: menor contacto del personal de salud con el paciente, aumento de la ansiedad y depresión, disminución de la satisfacción de los cuidados recibidos y un alto número de eventos adversos ${ }^{1-3}$. En Chile no existen reportes al respecto.

Comentario: Si bien, se ha asociado la precaución de contacto con un mayor número de eventos adversos, dada la fuerza de la recomendación de esta medida en prevenir la transmisión de microorganismos multirresistentes, resulta importante contrastar estos riesgos con los beneficios de su indicación. Por lo pronto, se hace necesario considerar esta medida como un riesgo adicional para los pacientes hospitalizados en UPC, debiendo vigilarse en forma estricta la ocurrencia de estos eventos.

\section{Referencias bibliográficas}

1.- Abad C, Fearday A, Safdar N. Adverse effects of isolation in hospitalised patients: a systematic review. J Hosp Infect 2010; 76: 97-102.

2.- Morgan D J, Diekema D J, Sepkowitz K, Perencevich E N. Adverse outcomes associated with contact precautions: A review of the literature. Am J Infect Control 2009; 37: 8593.

3.- Mehrotra P, Croft L, Day H, Perencevich E N. Effects of contact precautions on patient perception of care and satisfaction: a prospective cohort study. Infect Control Hosp Epidemiol 2013; 34: 1087-93.

Alejandra Zambrano Comité IAAS Red Salud UC-CHRISTUS Comité Consultivo IAAS Sochinf azambra@med.puc.cl 